\title{
UTILIDAD DEL LÁSER EN EL TRATAMIENTO DE LAS ESTENOSIS URETRALES
}

Jesús Moreno Sierra, Sara Prieto Nogal, Isabel Galante Romo, Lorena Fernández Montarroso, Juan Carlos López Corral y Ángel Silmi Moyano.

Servicio de Urología. Hospital Clínico San Carlos. Universidad Complutense. Madrid. España.

\begin{abstract}
Resumen.- INTRODUCCIÓN: La uretrotomía óptica fue introducida por Sachse (1) en 1973 y se ha comprobado una tasa de recurrencia de 75-80\% a largo plazo. Esto estimuló la búsqueda de nuevas terapias con una menor incidencia de recurrencia. Se intentaron varios tipos de láser: Nd:YAG, KTP, Argón, Ho:YAG, diodo... Desde finales de los 70 diferentes tipos de láser se han venido utilizando en el tratamiento de las estenosis uretrales.
\end{abstract}

OBJETIVO: Describir la utilidad de la energia láser en las estenosis uretrales, fundamentalmente recurrentes y analizar la experiencia existente con los diferentes tipos de láser (diodo, Nd:Yag, Holmium, KTP, Argon, etc).
MÉTODOS: Hemos realizado una revisión sistemática de la bibliografía, basada en una búsqueda en Medline y análisis detallado de los artículos seleccionados.

CONCLUSIONES: 11. El uso del láser en el tratamiento de las estenosis uretrales se revela por el momento como una alternativa válida, eficaz y segura, al menos a medio plazo, a la uretrotomía óptica; sin embargo por el momento no se ha demostrado que sea mejor que esta. 2). La elección del tratamiento es cirujano dependiente, y ninguna técnica ha mostrado claramente ser superior a las demás. 3). Se precisan estudios prospectivos a largo plazo, con un mayor número de pacientes y con mayor seguimiento. 4). La tecnología láser es cara y no está disponible en todos los centros.

Palabras clave: Estenosis de uretra recurrente. Láser.

Summary.- INTRODUCTION: Optical urethrotomy was introduced by Sachse (1) in 1973 and it has a registered longterm recurrence rate of $75-80 \%$. This stimulated the search for new therapies with less recurrences. Several types of láser were tried: Nd:YAG, KTP, Argon, Ho:YAG, diode,... Since the end of the '7Os various types of láser are being used for the treatment of ureteral stenosis.

OBJECTIVES: To describe the usefulness of the láser energy in the treatment of ureteral stenosis, mainly recurrent stenosis and to analyze the current experience with various types of láser (diode, nd:yag, holmium, argon,...)

METHODS: We performed the systematic review of the bibliography, based on a medline search, and a detailed analysis of the selected articles.

CONCLUSIONS: 11 the use of láser in the treatment of urethral stenosis is on the a valid, effective, and safe alternative option to optical urethrotomy, at least in the mid term; nevertheless, it has not demonstrated to date being better than that. 21 
The election of treatment is surgeon dependent and, and no single technique has demonstrated to be clearly better than the others. 3) Prospective long-term studies with larger numbers of patients and longer follow-up are necessary. 4) láser technology is extensive and it is not available in all centers.

Keywords: Recurrent urethral stenosis. Laser.

\section{INTRODUCCIÓN}

La elección de un adecuado tratamiento en pacientes con estenosis uretral depende de múltiples factores y en la actualidad no es fácil. La morbilidad de la cirugía uretral abierta es alta y con un elevado índice de recurrencias. Esto obligó a idear nuevas soluciones y en este sentido el abordaje endoscópico surgió como un método menos traumático y con una menor morbilidad.

De una forma resumida podemos considerar que el "patrón de oro" en las estenosis cortas es la uretrotomía óptico con corte frío. En 1795, Philip Syng fue el primero en realizar una uretrotomía interna usando un uretrotomo. Desde entonces se han descrito un sin fin de mejoras y adelantos que han llevado a la actual uretrotomía óptica, que inicialmente fue introducida por Sachse (1) en 1973, aunque por desgracia la tasa de recurrencia a largo plazo es elevada (75-80\%). En la actualidad disponemos de diferentes opciones para realizar la incisión endoscópica uretral: corte frío, electrotomía y la energía láser. Sea como fuere, la estenosis de uretra es una patología que aún no está resuelta y el gran caballo de batalla es la recurrencia.

En un intento de aportar nuevas soluciones a esta problemática, desde finales de los años 70 se ha aplicado la energía láser (Nd:YAG, KTP, Argón, Ho:YAG, diodo, etc) como una nueva forma de tratar la estenosis de uretra recurrente. Las heridas hechas con láser curan con menos cicatriz que aquellas realizadas con electrocauterio $o$ con corte frío, lo que explicaría la baja tasa de recurrencia. La principal diferencia entre el corte con láser y la uretrotomía clásica es que con láser no se corta ni separa el tejido, sino que el tejido enfermo se evapora con un insignificante efecto provocado por el calor sobre los tejidos profundos.

En la Tabla I pueden observarse las diferencias existentes entre los diferentes tipos de cortes, según las diferentes fuentes de energía utilizadas.

El uso del láser en estenosis uretrales no obstructivas se hace en la esperanza de vaporizar el tejido cicatricial y reducir la incidencia de reestenosis. La clave del éxito del láser no es sólo incidir el duro tejido fibrótico, sino hacer desaparecer la cápsula fibrótica pétrea para prevenir la reestenosis en el lugar.

\section{BASES FÍSICAS DE LA ENERGIA LÁSER}

Láser (Light Amplification by Stimulated Emission of Radiation), significa: "Amplificación de Luz por Emisión Estimulada de Radiación". La eficiencia de cada tipo de láser para vaporizar tejido y la profundidad de su efecto coagulativo dependen de su longitud de onda, de su modo de operación (continuo o pulsátil) y de la potencia de esta fuente de energía.

La luz láser se utiliza como herramienta quirúrgica porque sus efectos térmicos y mecánicos sobre el tejido son debidos a sus características físicas. La luz monocromática, es decir, una luz con un color único definido por su longitud de onda particular, puede utilizarse para actuar selectivamente sobre un tejido o una estructura dentro de un tejido que absorbe específicamente la luz de una cierta longitud de onda. La luz colimada, que viaja en un haz de fotones paralelos, puede ser conducida por fibra óptica y enfocada en un punto pequeño. Esta propiedad permite la aplicación de calor inducido por el láser para destruir un tejido con gran precisión. Al dirigir la luz monocromática y colimada contra el tejido parte de esta se refleja parcialmente en la superficie. La luz restante penetra en el tejido y se absorbe en parte por un componente del tejido que actúa como cromóforo para esa longitud de onda en particular, y en parte se disipa de forma difusa por la falta de homogeneidad del tejido. Ambos fenómenos, conocidos como absorción y disipación, limitan la profundidad a la que penetra la luz láser. Esa profundidad se conoce como profundidad de penetración óptica.

TABLA I. DIFERENCIAS EXISTENTES ENTRE LOS DIFERENTES TIPOS DE CORTES, SEGÚN LAS DIFERENTES FUENTES DE ENERGÍA UTILIZADAS.

\begin{tabular}{|l|c|c|c|}
\hline & CORTE FRIO & ELECTROCAUTERIO & LÁSER \\
\hline BASE FISICA DEL CORTE & Incisión & Necrosis coagulativa y fibrosis & Vaporización de tejido cicatricial \\
\hline CICATRIZ & + & + & - \\
\hline RECURRENCIA & + & + & - \\
\hline
\end{tabular}


La absorción de la luz láser en el tejido uretral está gobernada por dos componentes del mismo: el agua y la hemoglobina. La absorción de la luz láser genera calor en el tejido con la consecuente vaporización de su agua y la desnaturalización de sus proteínas, dependiendo de la temperatura local alcanza en las distintas zonas del área tratada. El calor se confina a un volumen muy pequeño de tejido superficial que se evaporiza inmediatamente, se induce un calentamiento rápido del tejido a grandes temperaturas $\left(>300^{\circ} \mathrm{C}\right)$. La ebullición del agua celular e intersticial provoca el estallido de las membranas celulares y la disrupción del intersticio tisular induciendo una vaporización muy eficiente. Los patrones de absorción de los láseres de distinta longitud de onda en agua y en hemoglobina son sustancialmente diferentes, lo que condiciona su profundidad de penetración y las lesiones a los tejidos circundantes (Tabla II) (2).

\section{TIPOS DE LÁSER UTILIZADOS EN ESTENOSIS DE URETRA}

\section{LÁSER DIODO}

Se trata de aparatos electrónicos semiconductores que emiten luz láser como corriente eléctrica que pasa a través de ellos. Los semiconductores están hechos de aluminio-galio arsénico y emiten un rayo láser con una longitud de onda que varía según el modelo.

La energía láser se transmite de manera eficiente a la uretra a través de una fibra óptica de cuarzo de 1.5 . $2 \mathrm{~m}$ de longitud, con un diámetro de 400-600 micras. Estas fibras son semirrígidas y pueden pasar fácilmente a través de los canales de cateterización de todos los cistoscopios, incluidos los pediátricos de $11 \mathrm{~F}$. Se utilizan guías de metal en vez de catéteres ureterales para tutorizar, ya que aquellos pueden quemarse con el calor producido por el láser.

La experiencia que que describe Kamal (3), utiliza el láser diodo con longitud de onda de $805 \mathrm{~nm}$ en 22 pacientes con estenosis de etiología diversa. El poder de alimentación fue de 18-20 W, usando el modo continuo. La técnica utilizada, no es una uretrotomía simple sino una completa extirpación del tejido fibroso de la estenosis llevado a cabo por contacto directo de la fibra de láser con el tejido, además de inducir vaporización. De esta forma se producen varios ojales, que posteriormente se conectan unos a otros hasta que todo el tejido fibroso de la estenosis se evapore por completo y pueda verse tejido uretral sano. La duración del procedimiento oscila entre 15 y 35 minutos, precisándose mantener un sondaje vesical de 1 a 5 días. Esta técnica puede usarse tanto como primera opción, como en estenosis recurrentes después de una uretrotomía óptica. La pérdida de sangre es mínima $(10 \mathrm{ml})$ y los resultados clínicos y flujométricos son buenos, describiéndose un $78.5 \%$ de éxitos en los pacientes en los que se utilizó como tratamiento de primera línea. En los casos de estenosis complicadas, con tratamientos previos, la tasa de recurrencia fue alta $(72.7 \%)$ pero el momento de aparición fue mucho más tardío que con el tratamiento endoscópico clásico.

Guazzieri (4) utiliza el modelo de longitud de onda de $980 \mathrm{~nm}$ y describe una serie de 45 pacientes, con una técnica quirúrgica similar a la uretrotomía óptica ya que realiza un corte a las 6 del horario endoscópico, con sangrado insignificante y buenos resultados: $79.5 \%$ de éxitos al año de seguimiento y $71 \%$ a los 2 años. El flujo máximo en esta serie oscila entre 15.5 y $18 \mathrm{ml} / \mathrm{min}$.

Al desaparecer todo el tejido fibroso las tasas de recurrencia son menores y cuando se producen son a más largo plazo. Comparativamente, el láser diodo ofrece algunas ventajas respecto al Nd:YAG: más barato, buena ventilación, mayor duración y facil uso en el campo quirúrgico.

El láser diodo es un método eficaz y fiable método para tratar estenosis uretrales, con una relativamente baja tasa de recurrencia. La pérdida mínima de sangre, corta estancia hospitalaria y la posibilidad de realizarlo con anestesia local son algunas de sus ventajas.

TABLA II. CARACTERÍSTICAS DE LOS DISTINTOS TIPOS DE LÁSER.

\begin{tabular}{|c|c|c|c|c|}
\hline Láser & $\begin{array}{c}\text { Longitud de } \\
\text { onda }(\mathbf{m m})\end{array}$ & $\begin{array}{c}\text { Penetración óptica } \\
\text { en agua }\end{array}$ & $\begin{array}{c}\text { Penetración óptica } \\
\text { en tejido }\end{array}$ & Comentarios \\
\hline Diodo & 830 & $30 \mathrm{~cm}$ & $5 \mathrm{~mm}$ & Necrosis coagulativa extensa \\
\hline Nd:YAG & 1064 & $8 \mathrm{~cm}$ & $10 \mathrm{~mm}$ & Necrosis coagulativa extensa \\
\hline Ho:YAG & 2100 & $0.4 \mathrm{~mm}$ & $0.4 \mathrm{~mm}$ & Vaporización poco intensa \\
\hline Ho:YAG contacto & 2100 & $0.4 \mathrm{~mm}$ & $0.4 \mathrm{~mm}$ & Prolongada curva de aprendizaje \\
\hline KTP & 532 & $30 \mathrm{~m}$ & $0.8 \mathrm{~mm}$ & Ambulatorio, fácil de usar \\
\hline
\end{tabular}




\section{LÁSER Nd:YAG}

El láser (Neodimio) Nd:YAG emite un rayo infrarrojo invisible con una longitud de onda de $1064 \mathrm{~nm}$. A esta longitud de onda el láser Nd:YAG se absorbe aproximadamente 200 veces menos por la hemoglobina que el láser KTP. Por lo tanto, al no verse atenuado por su absorción de la hemoglobina, el rayo de Nd:YAG penetra mas profundamente en el tejido (profundidad de penetración óptica de $10 \mathrm{~mm}$ ). La energía se difunde por un gran volumen de tejido que se calienta lentamente y tiene tiempo de difundirse a zonas profundas del tejido antes de que se produzca vaporización relativamente más lenta y superficial. Por lo tanto la vaporización producida por el láser del $\mathrm{Nd}$ : YAG es superficial y limitada, produciendo coagulación profunda. Esto condiciona la aparición de cierto edema postoperatorio en el tejido coagulado residual, y la posibilidad de retención urinaria transitoria, disuria ocasional y desprendimiento del tejido necrótico. Se ha usado con varios grados de resultados. Con el método de no contacto los resultados han sido decepcionantes, mientras que con el método de contacto, la tasa de recurrencia ha sido alta $(67 \%)$ con un año de seguimiento.

Perkash (5), usando el cincel de cristal por contacto, enroscado a una sonda de $2.5-3.5 \mathrm{~mm}$ en una fibra semirrígida, reporta un $93 \%$ de éxito con un seguimiento de 28.2 meses en 42 pacientes con estenosis de uretra de origen traumático y de $1 \mathrm{a} 4 \mathrm{~cm}$ de longitud. La técnica es por contacto, y realiza una vaporización circunferencial en los casos más simples y una técnica con ojales similar a la que se describía en el láser diodo en las más complejas.

Büllow (6) realizó el primer tratamiento de estenosis uretral con láser Nd:YAG, publicado en 1979, después de los experimentos realizados en uretras de perros. Dogra (7) reporta buenos resultados usando este láser, también mediante técnica de contacto, en estenosis pos-

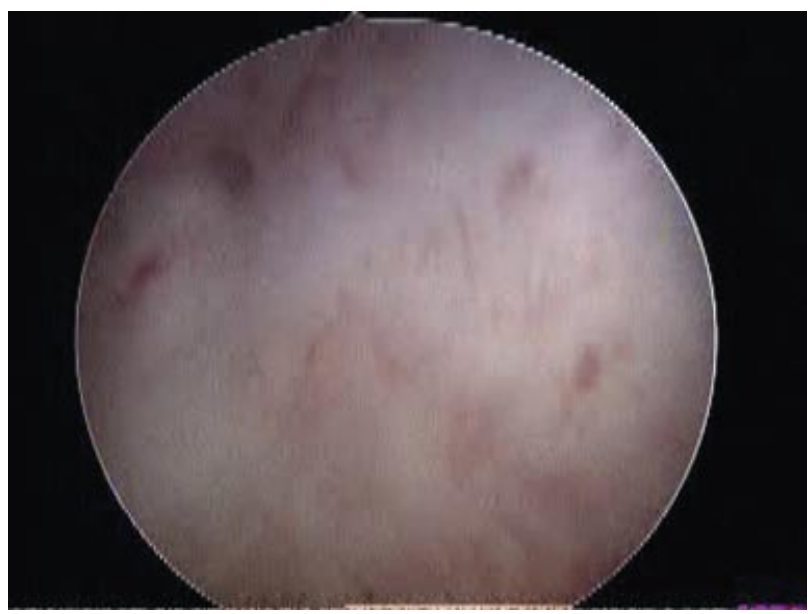

FIGURA 1. Estenosis de uretra bulbar puntiforme después de una fráctura pélvica en paciente de 64 años. No se pudo sondar y fue portador de talla vesical durante dos meses. traumáticas de la uretra bulbomembranosa. Inicialmente publicó una serie de 8 pacientes y ante los buenos resultados la amplió a 65 pacientes, con mayor período de seguimiento (8). En este trabajo se realiza una uretrografía anterógrada y otra retrógrada para ver in situ la estenosis completa de la uretra. La fibra es de 600 micras a 15-25 W con modo continuo y se utiliza una guía metálica de 20 Ch suprapúbica, que para encontrar el camino correcto y evitar falsas vías. El procedimiento se inicia dando un corte a las 12 y se profundiza hasta llegar a la sonda metálica, después se vaporiza circunferencialmente el tejido cicatricial blanquecino hasta llegar a tejido sano, moviendo la vaina de metal de un lado a otro por la zona estenótica, después se coloca una sonda vesical de 16-18 $\mathrm{Ch}$ de 4 a 6 semanas y se retira la talla suprapúbica. La pérdida de sangre es insignificante y la visión perfecta debido a la vaporización hemostática conseguida por la fibra del láser. El tiempo quirúrgico es de 45 a 70 minutos, con una media de 54 y se obtienen buenos resultados clínicos, uretrocistográficos y flujométricos. Dogra (9) también realiza esta técnica en niños, obteniendo también buenos resultados.

Utilizando una técnica de no contacto, Smith (10) publicó una serie de 24 pacientes tratados con láser $\mathrm{Nd}$ : YAG con punta de zafiro que incrementa la densidad de energía y el efecto de corte; sin embargo los resultados no fueron buenos ya que hasta un $67 \%$ recidivaron en menos de un año, por lo que no parece tener ninguna ventaja sobre la uretrotomía clásica.

Este láser es el utilizado de manera más frecuente para el manejo de estenosis uretrales por la facilidad de paso a través de endoscopios con fibra semirrígida pequeña. Se ha utilizado con técnicas de contacto y no contacto, cuando se usa la de no contacto, el efecto primario sobre el tejido es la coagulación térmica y el efecto de corte es limitado. La densidad de energía del láser se puede incre-

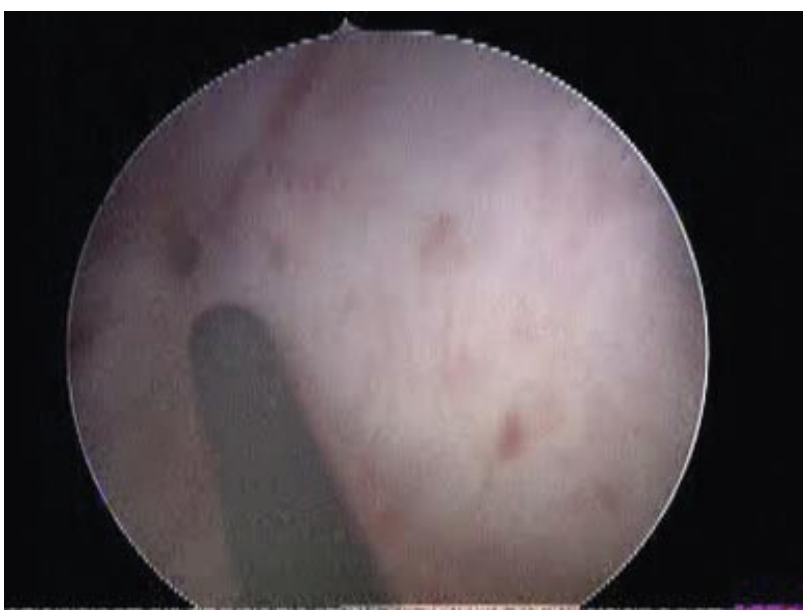

FIGURA 2. Guía de 0.035 situada en la proximidad de la estenosis uretral. 


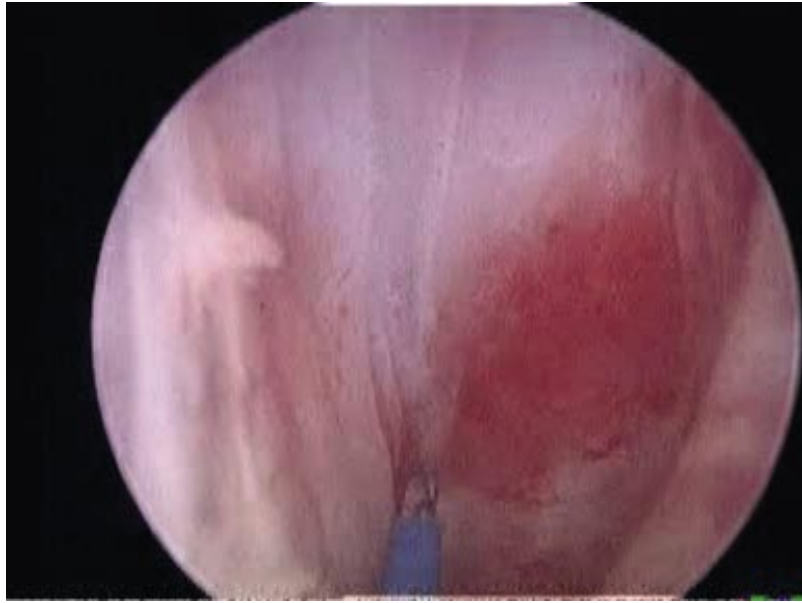

FIGURA 3. Inicio de la sección de la estenosis de uretra con láser Holmium utilizando una fibra de 365 micras.

mentar poniendo en contacto la fibra con el tejido, esta alta concentración de energía en un pequeño área de tejido, incrementa la vaporización del tejido y el efecto de corte, haciendo ideal para la incisión y vaporización circunferencial de las estenosis uretrales. A pesar de que las series son pequeñas y el tiempo de seguimiento corto, los resultados son esperanzadores.

\section{LÁSER (Holmium) Ho:YAG}

Las cualidades de este láser cumplen todos los requisitos. Combina las cualidades del Nd:YAG y del dióxido de carbono, realizando corte y coagulación al mismo tiempo. Emite una longitud de onda de $2100 \mathrm{~nm}$, que presenta una gran absorción en el agua lo que resulta en un muy

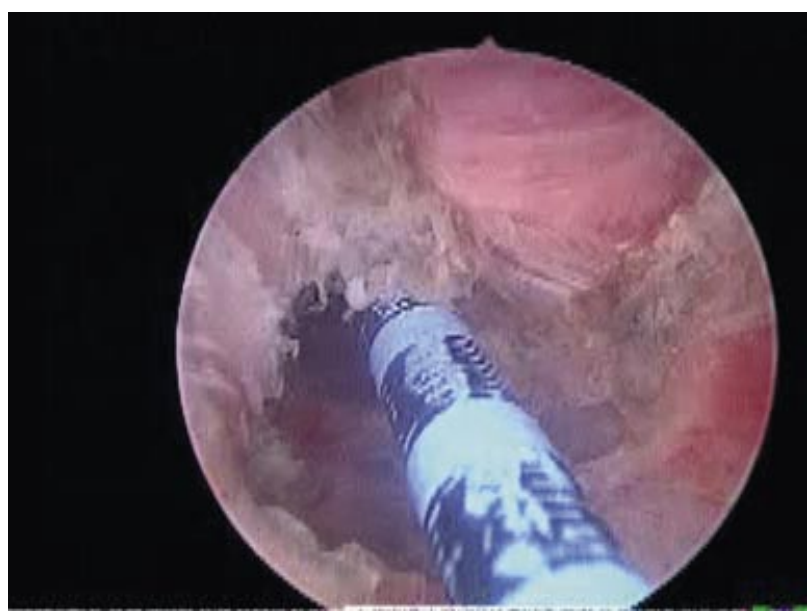

FIGURA 5. Resultado final de la sección endoscópica con láser Holmium de la estenosis uretral.

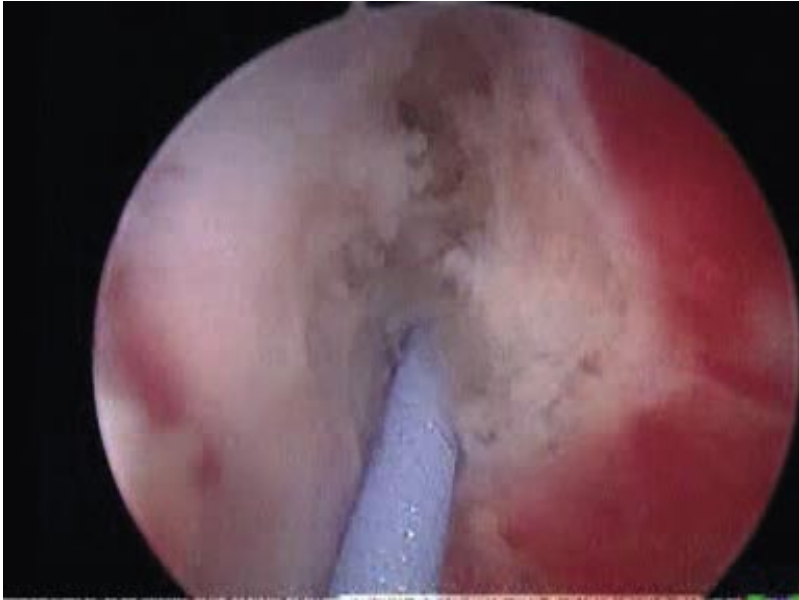

FIGURA 4. Imagen endoscópica del callo fibrótico uretral seccionado con láser Holmium.

preciso y superficial corte/penetración $(0.5 \mathrm{~mm})$, (esto lo diferencia y mejora de otros láseres) lo que reduce el riesgo de lesión térmica al tejido de alrededor y por consiguiente la cicatriz. Este láser calienta el agua de irrigación y crea una burbuja de vapor. El impacto mecánico pulsátil producido por las burbujas creadas secuencialmente rompe la superficie del tejido. Esto permite a este láser funcionar como herramienta de corte si se utiliza en contacto directo con el tejido.

Sus ventajas son las siguientes: corte preciso, ablación tisular, hemostasia (resulta en una mejor visibilidad durante el procedimiento) y soldado de tejidos (lo que previene la extravasación del líquido de irrigación). Puede usarse con el uretrotomo, el cistoscopio pediátrico o con un ureteroscopio rígido, en función del calibre de la ure-

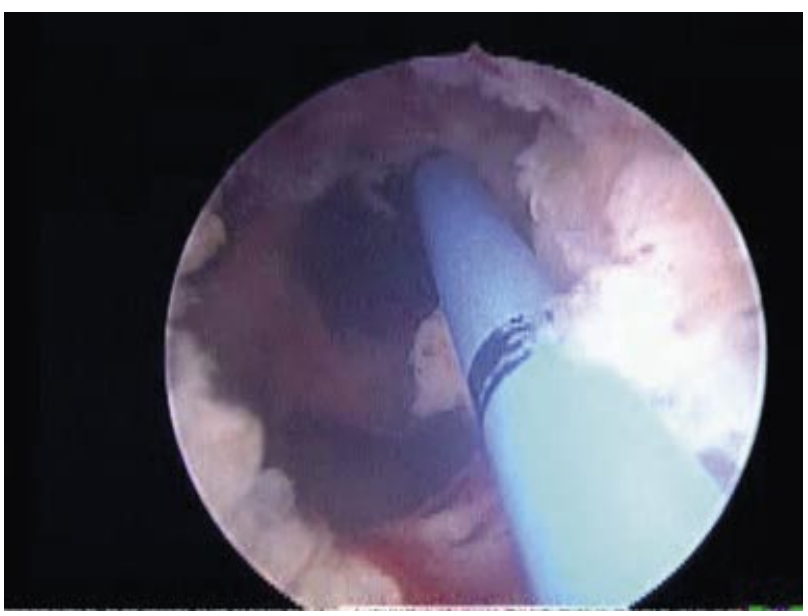

FIGURA 6. Resultado final del tratamiento de la estenosis de uretra con láser Holmium. El paciente tiene una excelente calidad miccional a los dos meses de la intervención. 
tra. Las fibras de cuarzo utilizadas pueden ser terminales - laterales, siendo estas últimas las más idóneas para el uso quirúrgico. Además también puede utilizarse con fibra óptica por su longitud de onda, lo que le hace ideal para la endourología. De hecho, es el láser más utilizado en distintos procedimientos endourológicos: litofragmentación, resección de tumores vesicales, tratamiento de estenosis ureterales y uretrales, resección prostática, etc.

Dogra (11) ha publicado una serie de 29 pacientes con estenosis uretral menores de $2.5 \mathrm{~cm}$, en la que realiza la uretrotomía creando un orificio central en el tejido fibrótico de la estenosis usando el láser holmiumYAG (yttrium aluminium garnet) con la fibra de 365 micras activada a 15-30 W, luego pasa una guía a través del orificio hasta la vejiga, a continuación hace tres incisiones a las 12, 4 y 8 horas del horario endoscópico desde el orificio creado inicialmente, seguidamente vaporizamos el tejido cicatricial entre las tres incisiones resultando un canal uretral amplio. Utiliza una guía metálica suprapúbica tal y como describía también en su serie del láser $\mathrm{Nd}$ :YAG, dejando la sonda vesical durante 6 semanas. Los resultados son esperanzadores, con un éxito total del $65.5 \%$ y aceptable en un $31.03 \%$ (Figuras 1, 2, 3, 4, 5 y 6).

Matsuoka (12) varía la técnica realizando un corte anterógrado utilizando un ureteroscopio semirrígido, a las 2 y las 10 del horario endoscópico, como publicó en una serie de 28 pacientes. Obtuvo buenos resultados, con un éxito del $74 \%$. Recomienda esta técnica para estenosis más largas de $1.5-2 \mathrm{~cm}$.

Kamp (13) describe su experiencia con el Ho:YAG de baja intensidad en un grupo de 32 pacientes, usando una energía láser de 1200 a 1400 mJ y una frecuencia de 10 a $13 \mathrm{~Hz}$. Realiza una incisión a las 12 del horario endoscópico, o en el punto más estrecho en las estenosis irregulares y a partir de aquí vaporiza el resto de la cicatriz. Tan sólo mantiene la sonda vesical 4 días, instilando previo a la retirada triamcinolona; obteniendo buenos resultados: éxito de $75 \%$, sin complicaciones intraoperatorias y con un tiempo de seguimiento medio de 27 meses.

Lagerveld (14) usa el Ho:YAG para estenosis de la anastomosis uretrovesical postprostatectomía radical, en una serie de 10 pacientes, realizando una incisión a las 6 horas del horario endoscópico y vaporizando toda la cicatriz entre las 3 y las 9, con buen resultado en todos los pacientes.

Se han descrito otras técnicas con este láser, como por ejemplo la publicada por Thomas (15) en la que utiliza una fibra de láser como guía para penetrar en la estenosis, con buenos resultados pero con un coste adicional cuantioso. Se ha utilizado también en pacientes pediátricos, igualmente con buenos resultados (16).

En todas las series el tiempo quirúrgico y la estancia hospitalaria son menores que los requeridos para la uretrotomía óptica clásica, se obtienen buenos resultados en estenosis menores de $2.5 \mathrm{~cm}$, tratandose de un método fácil, efectivo y seguro, aunque se necesitan más estudios con mayor tiempo de seguimiento para poder sacar conclusiones.

\section{LÁSER KTP}

La fuente de luz láser KTP (Potassium Titanyl Phosphate) emite luz colimada y coherente visible, de color verde, con una longitud de onda que corresponde exactamente a la del láser Nd:YAG $(532 \mathrm{~nm})$. Esta luz verde es absorbida con gran afinidad por los tejidos rojos, es decir, por los tejidos con alto contenido en oxihemoglobina, por lo que cualquier órgano bien vascularizado será un objetivo excelente para este tipo de láser.

La energía del láser KTP prácticamente no se absorbe en el agua y es capaz de atravesar este medio sin atenuarse, lo que hace que este láser sea idóneo para vaporizar tejidos en un medio acuoso. La penetración óptica de este láser en el tejido es muy limitada $(0.8 \mathrm{~mm})$, lo que significa que prácticamente toda la energía se deposita en la superficie del tejido, disipándose muy poco calor al plano tisular inmediatamente inferior. El uso de este láser se ha desarrollado más para el tratamiento de la HBP que para el de las estenosis uretrales aunque ya en 1988 se utilizaba para este fin.

Uno de los primeros en utilizarlo fue Shanberg (17) en una serie de 20 pacientes con estenosis uretrales recidivadas tras otros tratamientos quirúrgicos, obteniendo buenos resultados (68.2\%) a pesar de ser la primera serie publicada.

Turek (18) publicó un trabajo en el que trataba las estenosis uretrales de diferentes etiologías con láser KTP532 , con un éxito total del $59 \%$, éxito parcial de $20.5 \%$ (síntomas sin estenosis) y otro $20.5 \%$ precisaron nuevamente cirugía. La técnica utilizada fue la ablación circunferencial del tejido estenótico.

Schmidlin (19) publicó otro trabajo de las mismas características, obteniendo un éxito de $81 \%$ y sólo un $19 \%$ precisaron de nuevo tratamiento quirúrgico. En ninguno de los dos tuvieron complicaciones intra ni perioperatorias, manteniéndose los resultados a medio plazo. Dada su precisión se ha utilizado también en las estenosis uretrales en niños. El láser KTP se muestra como una alternativa válida, eficaz y segura para el tratamiento de la estenosis uretral pero los estudios realizados hasta el momento no disponen de series ampliar y su seguimiento es escaso a medio plazo.

\section{LÁSER ARGON}

Las características físicas de la longitud de onda del láser argón permiten una incisión precisa con excelente hemostasia y daños colaterales mínimos sobre los tejidos circundantes. Se utiliza de manera similar al uretrotomo ya que realiza una incisión hemostática que reduce la necesidad de fulguración extensa del tejido lo que disminuye la tendencia de la formación de más tejido cicatricial. Se puede utilizar con anestesia local y sin catéter uretral postoperatorio (20).

Usado también por Becker (21) que consiguió unos datos similares a la uretrotomía convencional sin conseguir demostrar ventajas sobre los demás. 


\section{OTROS TIPOS DE LÁSER}

En los últimos 20 años se han desarrollado más tipos de láser que los ya comentados, pero con escasa repercusión, como por ejemplo el láser de $\mathrm{CO}_{2}$ (22), el láser excimer (23) y el Erbium:YAG que tanto a nivel in vivo como in Vitro puede considerarse una alternativa prometedora al Ho:YAG. Sin embargo, no han tenido apenas relevancia y prácticamente se han abandonado los estudios salvo con este último.

\section{CONCLUSIONES}

1. El uso del láser en el tratamiento de las estenosis uretrales es una alternativa válida, eficaz y segura, a la uretrotomía óptica; sin embargo por el momento no se ha demostrado que sea mejor que esta.

2. La elección del tratamiento es cirujano dependiente, y ninguna técnica ha mostrado claramente ser superior a las demás.

3. Se precisan estudios prospectivos a largo plazo, con un mayor número de pacientes y con mayor seguimiento

4. La tecnología láser es cara y no esta disponible en todos los centros.

\section{AGRADECIMIENTOS}

Fotos cortesía del Dr. Álvaro Serrano.

\section{BIBLIOGRAFÍA Y LECTURAS RECOMENDADAS (*lectura de interés $y^{* *}$ lectura fundamental)}

1. SACHSE, H.: "Zur Behandlung der Harnrohrenstriktur". Fortschr. Med., 92: 12, 1974.

**2. GÓMEZ SANCHA, F.: "Fotovaporización prostática con láser KTP”. Silmi Moyano, A y Moreno Sierra, J. Endourología, Laparoscopia y Terapia mínimamente invasiva en Urología, Clínicas Urológicas de la Complutense, 11: 879, 2005.

3. KAMAL, B.A.: "The use of the diode láser for treating uretrhal strictures". BJU Int., 87: 831, 2001.

*4. GUAZZIERI, S. y cols.: "980-nm diode láser treatment for recurrent urethral strictures". Eur. Urol., 39: 19, 2001.

5. PERKASH, I.: "Ablation of urethral strictures using contact chisel crystal firing neodymium: YAG láser". J. Urol., 157: 809, 1997.

6. BULLOW, H.; BULLOW, V.; FROHMULLER, H.G.W.: "Transurethral láser urethrotomy in men: Preliminary report". J. Urol., 121: 286, 1979.
*7. DOGRA, P.N.; ARON, M.; RAJEEV, T.P.: "Core trhough urethrotomy with the neodymium: YAG láser for posttraumatic obliterative strictures of the bulbomembranous urethra”. J. Urol., 161: 81, 1999.

8. DOGRA, P.N.; NABI, G.: "Core trhough urethrotomy using the neodymium: YAG láser for obliterative urethral strictures after traumatic urethral disruption and/or distraction defects: Long term outcome”. J. Urol., 167: 543, 2002.

9. DOGRA, P.N.; NABI, G.: "Nd: YAG láser core-through urethrotomy in obliterative posttraumatic urethral strictures in children". Pediatr. Surg. Int., 19: 652, 2003.

10. SMITH, J.A. Jr.: "Treatment of benign urethral strictures using a sapphire tipped neodymium: YAG láser". J. Urol., 142: 1221,-1989.

*11. DOGRA, P.N.; ANSARI, M.S.; GUPTA, N.P. y cols.: "Holmium láser core through urethrotomy for traumatic obliterative strictures of urethra: Initial experience". Urology, 64: 232, 2004.

12. MATSUOKA, K.; INOUE, M.; IIDA, S. y cols.: "Endoscopic antegrade láser incision in the treatment of urethral stricture". Urology, 60: 968, 2002.

13. KAMP, S.; KNOLL, T.; OSMAN, M.M. y cols.: "Lowpower holmium: YAG láser urethrotomy for treatment of urethral strictures: functional outcome and quality of life". J. Endourol., 20: 38, 2006.

*14. LAGERVELD, B.W.; LAGUNA, M.P.; DEBRUYNE, F.M. y cols.: "Holmium: YAG láser for treatment of strictures of vesicourethral anastomosis after radical prostatectomy". J. Endourol., 19: 497, 2005.

15. THOMAS, M.A.; ONG, A.M.; PINTO, P.A. y cols.: "Management of obliterated urinary segments using a láser fiber for access". J. Urol., 169: 2284, 2003.

16. FUTAO, S.; WENTONG, Z.; YAN, Z.: “Application of endoscopic Ho: YAG láser incision technique treating urethral strictures and urethral atresias in pediatric patients". Pediatr. Surg. Int., 22: 514, 2006.

*17. SHANBERG, A.; BAGHDASSARIAN, R.; TANSEY, L. y cols.: "KTP 532 láser in treatment of urethral strictures". Urology, 32: 517, 1988.

18. TUREK, P.J.; MALLOY, T.R.; CENDROM, M. y cols.: "KTP-532 láser ablation of urethral strictures". Urology, 40: 330, 1992.

*19. SCHMIDLING, F.; OSWALD, M.; ISELIN, C. y cols.: "Vaporization of urethral stenosis using the KTP 532 láser”. Ann. Urol., 31: 38, 1997.

20. ADKINS, W.C.: "Argon láser treatment of uretrhal stricture and vesical neck contracture". Láser Surg. Med., 8: 600, 1988.

**21. BECKER, H.C.; MILER, J.; NOSKE, H.D. y cols.: "Transurethral láser urethrotomy with the Argon láser: Experience with 900 urethrotomies in 450 patients from 1978 to 1993". Urol. Int., 55: 150,1995.

**22. McNICHOLAS, T.A.; COLLES, J.; BROWN, S.G. y cols.: "Treatment of urethral strictures with a prototype CO2 láser endoscope". Láser Surg. Med., 3: 427, 1988.

23. BAUR, H.; SCHNEIDER, W.; ATTWEIN, J.E.: "Treatment of recurrent urethral strictures by photoablation with Excimer láser". Baus Abstracts Book, pág. 118, 1992. 